\title{
SOME PROBLEMS IN INTERPOLATION BY CHARACTER- ISTIC FUNCTIONS OF LINEAR DIFFERENTIAL SYSTEMS OF THE FOURTH ORDER
}

\author{
KATHARINE E. O'BRIEN
}

In this paper we consider the convergence to $f(x)$, defined on $[0,1]$, of

$$
\Sigma_{p}[f(x)]=\alpha_{0 p} u_{0}(x)+\alpha_{1 p} u_{1}(x)+\cdots+\alpha_{p p} u_{p}(x),
$$

where $u_{n}(x),(n=0,1, \cdots, p)$, are characteristic functions of certain self-adjoint linear differential systems of fourth order,

$$
\alpha_{n p}=\sum_{k=0}^{p} f\left(x_{k}\right) u_{n}\left(x_{k}\right)\left\{\sum_{k=0}^{p} u_{n}^{2}\left(x_{k}\right)\right\}^{-1}, \quad n=0,1, \cdots, p,
$$

and the symbol $\sum^{\prime}$ is used in the sense $\sum_{k=0}^{p} y_{k}=y_{0} / 2+\sum_{k=1}^{p} y_{k}$. Throughout the discussion, $x_{k}=2 k /(2 p+1),(k=0,1, \cdots, p)$. The differential systems considered are

$$
u^{(\text {iv })}-\rho^{4} u=0,
$$

with boundary conditions

I. $u^{\prime}(0)=0, u^{\prime \prime \prime}(0)=0, u^{\prime}(1)=0, u^{\prime \prime \prime}(1)+u(1)=0$,

II. $u^{\prime}(0)=0, u^{\prime \prime \prime}(0)=0, u^{\prime}(1)+u(1)=0, u^{\prime \prime \prime}(1)+u^{\prime \prime}(1)=0$,

III. $u(0)=0, u^{\prime \prime}(0)=0, u(1)=0, u^{\prime \prime}(1)+u^{\prime}(1)=0$,

IV. $u^{\prime}(0)=0, u^{\prime \prime \prime}(0)=0, u(1)=0, u^{\prime \prime}(1)+u^{\prime}(1)=0$,

V. $u(0)=0, u^{\prime}(0)=0, u(1)=0, u^{\prime}(1)=0$,

VI. $u^{\prime}(0)=0, u^{\prime \prime \prime}(0)=0, u(1)=0, u^{\prime}(1)=0$.

The following theorems may be proved for these systems respectively.

I, II. If $f(x)$ is continuous and of bounded variation in $[0,1]$, then $\lim _{p \rightarrow \infty} \Sigma_{p}[f(x)]=f(x)$ uniformly in $[0,1]$.

III. If $f(x)$ is continuous and of bounded variation in $[0,1]$ and $f(0)=f(1)=0$, then $\lim _{p \rightarrow \infty} \Sigma_{p}[f(x)]=f(x)$ uniformly in $[0,1]$.

IV. If $f(x)$ is continuous and of bounded variation in $[0,1]$ and $f(1)=0$, then $\lim _{p \rightarrow \infty} \Sigma_{p}[f(x)]=f(x)$ uniformly in $[0,1-\eta]$.

V, VI. If $f(x)$ satisfies a Lipschitz condition in $[0,1]$ and $f(0)=f(1)$ $=0$, then $\lim _{p \rightarrow \infty} \Sigma_{p}[f(x)]=f(x)$ uniformly in $[\eta, 1-\eta]$.

Here and hereafter $\eta>0$ is arbitrarily small but fixed.

The method of proof for these theorems, as well as for those to fol- 
low, is essentially the same as that of C. M. Jensen [1], who considered convergence properties of a somewhat similar sum using Sturm-Liouville functions.

The following equivalence theorems (the term "equivalence" is used in the sense of Jensen) may be proved for systems I; II, III, IV, respectively.

I, II. If $f(x)$ is continuous in $[0,1]$, then

$$
\lim _{p \rightarrow \infty}\left\{\Sigma_{p}[f(x)]-T_{p}[f(x)]\right\}=0
$$

uniformly in $[0,1]$, where $T_{p}[f(x)]$ is the cosine interpolation formula

$$
\begin{aligned}
T_{p}[f(x)] & =a_{0 p}+a_{1 p} \cos \pi x+\cdots+a_{p p} \cos p \pi x, \\
a_{n p} & =\sum_{k=0}^{p} f\left(x_{k}\right) \cos n \pi x_{k}\left\{\sum_{k=0}^{p} \cos ^{2} n \pi x_{k}\right\}^{-1} \\
& = \begin{cases}\frac{4}{2 p+1} \sum_{k=0}^{p} f\left(x_{k}\right) \cos n \pi x_{k}, & n=1,2, \cdots, p, \\
\frac{2}{2 p+1} \sum_{k=0}^{p} f\left(x_{k}\right), & n=0 .\end{cases}
\end{aligned}
$$

III. If $f(x)$ is continuous in $[0,1]$, then

$$
\lim _{p \rightarrow \infty}\left\{\Sigma_{p}[f(x)]-\bar{T}_{p}[f(x)]\right\}=0
$$

uniformly in $[\eta, 1-\eta]$, where $\bar{T}_{p}[f(x)]$ is the sine interpolation formula

$$
\begin{aligned}
\bar{T}_{p}[f(x)] & =\bar{a}_{1_{p}} \sin \pi x+\bar{a}_{2 p} \sin 2 \pi x+\cdots+\bar{a}_{p p} \sin p \pi x, \\
\bar{a}_{n p} & =\sum_{k=0}^{p} f\left(x_{k}\right) \sin n \pi x_{k}\left\{\sum_{k=0}^{p} \sin ^{2} n \pi x_{k}\right\}^{-1} \\
& =\frac{4}{2 p+1} \sum_{k=0}^{p} f\left(x_{k}\right) \sin n \pi x_{k}, \quad n=1,2, \cdots, p .
\end{aligned}
$$

IV. If $f(x)$ is continuous in $[0,1]$, then

$$
\lim _{p \rightarrow \infty}\left\{\Sigma_{p}[f(x)]-U_{p}[f(x)]\right\}=0
$$

uniformly in $[\eta, 1-\eta]$, where $U_{p}[f(x)]$ is given by

$$
\begin{aligned}
U_{p}[f(x)]= & b_{0 p} \cos (\pi / 2) x+b_{1 p} \cos (3 \pi / 2) x+\cdots \\
& +b_{p p} \cos (p+1 / 2) \pi x \\
b_{n p}= & \sum_{k=0}^{p} f\left(x_{k}\right) \cos (n+1 / 2) \pi x_{k}\left\{\sum_{k=0}^{p} \cos ^{2}(n+1 / 2) \pi x_{k}\right\}^{-1}
\end{aligned}
$$




$$
= \begin{cases}\frac{4}{2 p+1} \sum_{k=0}^{p} f\left(x_{k}\right) \cos (n+1 / 2) \pi x_{k}, n=0,1, \cdots, p-1, \\ \frac{2}{2 p+1} \sum_{k=0}^{p} f\left(x_{k}\right) \cos (n+1 / 2) \pi x_{k}, & n=p .\end{cases}
$$

The first set of theorems also holds using characteristic functions of $u^{(\mathrm{iv})}-\left[\rho^{4}+\lambda(x)\right] u=0$, with the above boundary conditions, $\lambda(x)$ being an arbitrary continuous function. Here, provided $0 u(x) \not \equiv 0$, the term $0 u(x) \sum_{k=0}^{\prime p} f\left(x_{k}\right)_{0} u\left(x_{k}\right)\left\{\sum_{k=0}^{\prime p} u^{2}\left(x_{k}\right)\right\}^{-1}$ is adjoined to $\Sigma_{p}[f(x)]$, where $o u(x)$ is the characteristic function corresponding to $\rho=0$. (For $\lambda(x) \equiv 0, \rho=0$ is not a characteristic number.)

We give here the proof of the theorem for system V. System V is the problem of lateral vibrations of an elastic homogeneous rod clamped at both ends [2]. We first state two lemmas.

LEMMA 1. If $f(x)$ is continuous and of bounded variation in $[0,1]$ and $f(0)=f(1)=0$, then $W_{p}[f(x)]$ and $w_{p}[f(x)]$ tend to the same limit $M(x)$ uniformly in $[\eta, 1-\eta]$ as $p \rightarrow \infty$, where

$$
\begin{aligned}
W_{p}[f(x)]= & c_{0 p}[\cos (\pi / 2) x-\sin (\pi / 2) x] \\
& +c_{1 p}[\cos (3 \pi / 2) x-\sin (3 \pi / 2) x]+\cdots \\
& +c_{p p}[\cos (p+1 / 2) \pi x-\sin (p+1 / 2) \pi x], \\
c_{n p}= & \frac{2}{2 p+1} \sum_{k=0}^{p} f\left(x_{k}\right)\left[\cos (n+1 / 2) \pi x_{k}-\sin (n+1 / 2) \pi x_{k}\right], \\
& n=0,1, \cdots, p,
\end{aligned}
$$$$
w_{p}[f(x)]=c_{0}[\cos (\pi / 2) x-\sin (\pi / 2) x]+c_{1}[\cos (3 \pi / 2) x-\sin (3 \pi / 2) x]
$$$$
+\cdots+c_{p}[\cos (p+1 / 2) \pi x-\sin (p+1 / 2) \pi x] \text {, }
$$$$
c_{n}=\int_{0}^{1} f(t)[\cos (n+1 / 2) \pi t-\sin (n+1 / 2) \pi t] d t
$$$$
n=0,1, \cdots, p \text {. }
$$

LEMma 2. If $f(x)$ satisfies a Lipschitz condition in $[0,1]$ and $f(0)=f(1)=0$, then there exists a constant $C$, depending only on $\eta$, such that for $n$ sufficiently great, $p \geqq n$, and $x$ in $[\eta, 1-\eta]$,

$$
\left|\alpha_{n p} u_{n}(x)-c_{n p}[\cos (n+1 / 2) \pi x-\sin (n+1 / 2) \pi x]\right|<C / n^{2} .
$$

To prove Lemma 1, write

$$
\begin{aligned}
W_{p}[f(x)] & ={ }_{1} W_{p}[f(x)]+{ }_{2} W_{p}[f(x)]-{ }_{3} W_{p}[f(x)], \\
w_{p}[f(x)] & ={ }_{1} w_{p}[f(x)]+{ }_{2} w_{p}[f(x)]-{ }_{3} w_{p}[f(x)],
\end{aligned}
$$


where

$$
\begin{aligned}
{ }_{1} W_{p}[f(x)] & =\frac{2}{2 p+1} \sum_{k=0}^{p} f\left(x_{k}\right) \sum_{n=0}^{p} \cos (n+1 / 2) \pi x_{k} \cos (n+1 / 2) \pi x, \\
{ }_{2} W_{p}[f(x)] & =\frac{2}{2 p+1} \sum_{k=0}^{p} f\left(x_{k}\right) \sum_{n=0}^{p} \sin (n+1 / 2) \pi x_{k} \sin (n+1 / 2) \pi x, \\
{ }_{3} W_{p}[f(x)] & =\frac{2}{2 p+1} \sum_{k=0}^{p} f\left(x_{k}\right) \sum_{n=0}^{p} \sin (n+1 / 2) \pi\left(x_{k}+x\right), \\
{ }_{1} w_{p}[f(x)] & =\int_{0}^{1} f(t) \sum_{n=0}^{p} \cos (n+1 / 2) \pi t \cos (n+1 / 2) \pi x d t, \\
{ }_{2} w_{p}[f(x)] & =\int_{0}^{1} f(t) \sum_{n=0}^{p} \sin (n+1 / 2) \pi t \sin (n+1 / 2) \pi x d t, \\
{ }_{3} w_{p}[f(x)] & =\int_{0}^{1} f(t) \sum_{n=0}^{p} \sin (n+1 / 2) \pi(t+x) d t .
\end{aligned}
$$

We first show that if $f(x)$ is continuous and of bounded variation in $[0,1]$, then $\lim _{p \rightarrow \infty} 1 W_{p}[f(x)]=(1 / 2) f(x)$ uniformly in $[0,1-\eta]$. We employ the cosine interpolation formula $T_{p}[f(x)]$. We shall use $r_{n}(x)$ as generic notation for a function uniformly bounded in $n$ and for $x$ in $[0,1]$, unless the range for $x$ is otherwise stated, and $r_{n}\left[r_{n p}\right]$ for a quantity depending on $n[n$ and $p]$ and uniformly bounded in $n$ $[n$ and $p]$. We have

$$
\begin{aligned}
T_{p}[f(x)]= & \frac{2}{2 p+1} \sum_{k=0}^{p} f\left(x_{k}\right)+\frac{4}{2 p+1} \sum_{k=0}^{p} f\left(x_{k}\right) \sum_{n=1}^{p} \cos n \pi x_{k} \cos n \pi x \\
= & \frac{1}{2 p+1} \sum_{k=0}^{p} f\left(x_{k}\right)\left[\frac{\sin (p+1 / 2) \pi\left(x_{k}-x\right)}{\sin (\pi / 2)\left(x_{k}-x\right)}\right. \\
& \left.\quad+\frac{\sin (p+1 / 2) \pi\left(x_{k}+x\right)}{\sin (\pi / 2)\left(x_{k}+x\right)}\right] \\
= & \frac{1}{2 p+1} \sum_{k=0}^{p} f\left(x_{k}\right)\left[\frac{\sin p \pi\left(x_{k}-x\right) \cos (\pi / 2)\left(x_{k}-x\right)}{\sin (\pi / 2)\left(x_{k}-x\right)}\right. \\
& +\frac{2}{2 p+1} \cos p \pi x \sum_{k=0}^{p} f\left(x_{k}\right) \cos p \pi x_{k} .
\end{aligned}
$$


Using auxiliary Lemma A stated below, we have

$$
\begin{aligned}
T_{p}[f(x)]=\frac{1}{2 p+1} \sum_{k=0}^{p} f\left(x_{k}\right)\left[\frac{\sin p \pi\left(x_{k}-x\right) \cos (\pi / 2)\left(x_{k}-x\right)}{\sin (\pi / 2)\left(x_{k}-x\right)}\right. \\
\left.+\frac{\sin p \pi\left(x_{k}+x\right) \cos (\pi / 2)\left(x_{k}+x\right)}{\sin (\pi / 2)\left(x_{k}+x\right)}\right]+\frac{r_{p}(x)}{p}
\end{aligned}
$$

and

$$
\begin{aligned}
{ }_{1} W_{p}[f(x)]= & \frac{2}{2 p+1} \sum_{k=0}^{p} f\left(x_{k}\right) \sum_{n=0}^{p-1} \cos (n+1 / 2) \pi x_{k} \cos (n+1 / 2) \pi x \\
& +r_{p}(x) / p \\
= & \frac{1}{2(2 p+1)} \sum_{k=0}^{p} f\left(x_{k}\right)\left[\frac{\sin p \pi\left(x_{k}-x\right)}{\sin (\pi / 2)\left(x_{k}-x\right)}\right. \\
& \left.\quad+\frac{\sin p \pi\left(x_{k}+x\right)}{\sin (\pi / 2)\left(x_{k}+x\right)}\right]+\frac{r_{p}(x)}{p} .
\end{aligned}
$$

Thus

$$
\begin{aligned}
& { }_{1} W_{p}[f(x)]-(1 / 2) T_{p}[f(x)] \\
& =\frac{1}{2(2 p+1)} \sum_{k=0}^{p} f\left(x_{k}\right)\left[\frac{1-\cos (\pi / 2)\left(x_{k}-x\right)}{\sin (\pi / 2)\left(x_{k}-x\right)} \sin p \pi\left(x_{k}-x\right)\right. \\
& \left.\quad+\frac{1-\cos (\pi / 2)\left(x_{k}+x\right)}{\sin (\pi / 2)\left(x_{k}+x\right)} \sin p \pi\left(x_{k}+x\right)\right]+\frac{r_{p}(x)}{p} \\
& =\frac{1}{2(2 p+1)} \sum_{k=0}^{p} f\left(x_{k}\right)\left[\tan (\pi / 4)\left(x_{k}-x\right) \sin p \pi\left(x_{k}-x\right)\right. \\
& \left.\quad+\tan (\pi / 4)\left(x_{k}+x\right) \sin p \pi\left(x_{k}+x\right)\right]+r_{p}(x) / p .
\end{aligned}
$$

By reason of the nature of Lemma $A$ and the fact that for $t$ in $[0,1]$ the functions $\tan (\pi / 4)(t-x)$ and $\tan (\pi / 4)(t+x)$ are of uniform bounded variation with respect to $x$ in $[0,1-\eta]$, we have ${ }_{1} W_{p}[f(x)]-(1 / 2) T_{p}[f(x)]=r_{p}(x) / p$ for $x$ in $[0,1-\eta]$. Hence [3] $\lim _{p \rightarrow \infty} 1 W_{p}[f(x)]=(1 / 2) f(x)$ uniformly in $[0,1-\eta]$.

Employing $t_{p}[f(x)]$, the partial sum of order $p$ in the Fourier cosine series,

$$
\begin{aligned}
& t_{p}[f(x)]= a_{0}+a_{1} \cos \pi x+\cdots+a_{p} \cos p \pi x, \\
& a_{n}=\frac{\int_{0}^{1} f(x) \cos n \pi x d x}{\int_{0}^{1} \cos ^{2} n \pi x d x}= \begin{cases}2 \int_{0}^{1} f(x) \cos n \pi x d x, n=1,2, \cdots, p, \\
\int_{0}^{1} f(x) d x, & n=0,\end{cases}
\end{aligned}
$$


we may show similarly that if $f(x)$ is continuous and of bounded variation in $[0,1]$, then $\lim _{p \rightarrow \infty} 1 w_{p}[f(x)]=(1 / 2) f(x)$ uniformly in $[0,1-\eta]$. Employing $\bar{T}_{p}[f(x)]$, the sine interpolation formula, and $\bar{t}_{p}[f(x)]$, the partial sum of order $p$ in the Fourier sine series,

$\bar{t}_{p}[f(x)]=\bar{a}_{1} \sin \pi x+\bar{a}_{2} \sin 2 \pi x+\cdots+\bar{a}_{p} \sin p \pi x$,

$$
\bar{a}_{n}=\frac{\int_{0}^{1} f(x) \sin n \pi x d x}{\int_{0}^{1} \sin ^{2} n \pi x d x}=2 \int_{0}^{1} f(x) \sin n \pi x d x, n=1,2, \cdots, p,
$$

we may likewise show that if $f(x)$ is continuous and of bounded variation in $[0,1]$ and $f(0)=f(1)=0$, then

$$
\lim _{p \rightarrow \infty}{ }_{2} W_{p}[f(x)]=(1 / 2) f(x), \quad \lim _{p \rightarrow \infty}{ }_{2} w_{p}[f(x)]=(1 / 2) f(x)
$$

uniformly in $[0,1-\eta]$. In connection with ${ }_{1} w_{p}[f(x)]$ and ${ }_{2} w_{p}[f(x)]$ we use Lemma B, stated below.

Finally, we show that if $f(x)$ is of bounded variation in $[0,1]$, then ${ }_{3} W_{p}[f(x)]$ and ${ }_{3} w_{p}[f(x)]$ both tend to $(1 / 2) \int_{0}^{1} f(t)\{\sin (\pi / 2)(t+x)\}^{-1} d t$ uniformly in $[\eta, 1-\eta]$. We have

$$
{ }_{3} w_{p}[f(x)]=\frac{1}{2} \int_{0}^{1} f(t) \frac{1-\cos (p+1) \pi(t+x)}{\sin (\pi / 2)(t+x)} d t .
$$

For $x$ in $[\eta, 1-\eta]$,

$$
\begin{aligned}
{ }_{3} w_{p}[f(x)]= & \frac{1}{2} \int_{0}^{1} \frac{f(t)}{\sin (\pi / 2)(t+x)} d t \\
& -\frac{1}{2} \int_{0}^{1} f(t) \frac{\cos (p+1) \pi(t+x)}{\sin (\pi / 2)(t+x)} d t .
\end{aligned}
$$

Effecting some trigonometric reductions on the second integrand and using Lemma $B$, we have

$$
{ }_{3} w_{p}[f(x)]=\frac{1}{2} \int_{0}^{1} \frac{f(t)}{\sin (\pi / 2)(t+x)} d t+\frac{r_{p}(x)}{p} .
$$

We may then prove that

$$
\lim _{p \rightarrow \infty} W_{p}[f(x)]=\frac{1}{2} \int_{0}^{1} \frac{f(t)}{\sin (\pi / 2)(t+x)} d t
$$


uniformly in $[\eta, 1-\eta]$, using the fact just established that

$$
\lim _{p \rightarrow \infty}{ }_{3} w_{p}[f(x)]=\frac{1}{2} \int_{0}^{1} \frac{f(t)}{\sin (\pi / 2)(t+x)} d t
$$

uniformly in $[\eta, 1-\eta]$, together with Lem ma C, stated below.

LEMMA A. If $f(x)$ is of bounded variation in $[0,1]$, then for $n=1,2, \cdots, 2 p$,

$$
\begin{aligned}
& \frac{1}{2 p+1}\left|\sum_{k=0}^{p} f\left(x_{k}\right) \cos n \pi x_{k}\right|=\frac{r_{n p}}{n}, \\
& \frac{1}{2 p+1}\left|\sum_{k=0}^{p} f\left(x_{k}\right) \sin n \pi x_{k}\right|=\frac{r_{n p}}{n} .
\end{aligned}
$$

Also

$$
\frac{1}{2 p+1}\left|\sum_{k=0}^{p} f\left(x_{k}\right) \sin (2 p+1) \pi x_{k}\right|=0 .
$$

LEMMA B. If $f(x)$ is of bounded variation in $[0,1]$, then for $n>0$,

$$
\left|\int_{0}^{1} f(x) \cos n \pi x d x\right|=\frac{r_{n}}{n}, \quad\left|\int_{0}^{1} f(x) \sin n \pi x d x\right|=\frac{r_{n}}{n} .
$$

LEMMA C. If $f(x)$ is of bounded variation in $[0,1]$, then for any preassigned $\epsilon>0$ there exists $Q$ such that for $p>q \geqq Q$ and $x$ in $[\eta, 1]$,

$$
\frac{2}{2 p+1}\left|\sum_{k=0}^{p} f\left(x_{k}\right) \sum_{n=q+1}^{p} \sin (n+1 / 2) \pi\left(x_{k}+x\right)\right|<\epsilon .
$$

Lemma 2 is proved by means of auxiliary Lemmas D, E, and A.

LEMMA D. For $n$ sufficiently great, and $p \geqq n$,

$$
\left[\sum_{k=0}^{p} u_{n}^{2}\left(x_{k}\right)\right]^{-1}=\frac{2}{2 p+1}\left(1+\frac{r_{n p}}{n}\right) .
$$

LEMma E. If $f(x)$ satisfies a Lipschitz condition in $[0,1]$ and if $f(0)=f(1)=0$, then for $n=1,2, \cdots, p$,

$$
\begin{gathered}
\frac{1}{2 p+1}\left|\sum_{k=0}^{p} f\left(x_{k}\right) \exp \left\{-(n+1 / 2) \pi x_{k}\right\}\right|=\frac{r_{n p}}{n^{2}}, \\
\frac{1}{2 p+1}\left|\sum_{k=0}^{p} f\left(x_{k}\right) \exp \left\{-(n+1 / 2) \pi\left(1-x_{k}\right)\right\}\right|=\frac{r_{n p}}{n^{2}} .
\end{gathered}
$$


The following asymptotic expression is known [4] for the characteristic functions of system V:

$$
\begin{aligned}
u_{n}(x)= & \cos (n+1 / 2) \pi x-\sin (n+1 / 2) \pi x \\
& +(-1)^{n} \exp \{-(n+1 / 2) \pi(1-x)\} \\
& -\exp \{-(n+1 / 2) \pi x\}+\exp \{-(n+1 / 2) \pi\} r_{n}(x) .
\end{aligned}
$$

Define

$$
\begin{aligned}
\sigma_{p}[f(x)] & =\alpha_{0} u_{0}(x)+\alpha_{1} u_{1}(x)+\cdots+\alpha_{p} u_{p}(x), \\
\alpha_{n} & =\frac{\int_{0}^{1} f(x) u_{n}(x) d x}{\int_{0}^{1} u_{n}^{2}(x) d x}, \quad n=0,1, \cdots, p .
\end{aligned}
$$

Denote by $\Sigma_{p}^{(r, s)}$ the sum of the terms in $\Sigma_{p}[f(x)]$ with subscripts $r$ to $s$ inclusive; similarly in the other sums.

For $x$ in $[\eta, 1-\eta]$,

$$
\begin{aligned}
\left|f(x)-\Sigma_{p}[f(x)]\right| \leqq & \left|\sigma^{(0, N)}-\Sigma_{p}^{(0, N)}\right|+\left|W_{p}^{(N+1, p)}-\Sigma_{p}^{(N+1, p)}\right| \\
& +\left|W_{p}^{(0, N)}-w^{(0, N)}\right|+\left|M(x)-W_{p}^{(0, p)}\right| \\
& +\left|w^{(0, N)}-M(x)\right|+\left|f(x)-\sigma^{(0, N)}\right| .
\end{aligned}
$$

The right-hand member can be made arbitrarily small by choosing $p$ sufficiently large. Call the six terms $D_{1}, D_{2}, \cdots, D_{6}$. Given $\epsilon$, first choose $N$ sufficiently large so that $D_{2}<\epsilon, D_{5}<\epsilon, D_{6}<\epsilon$, for $p \geqq N+1$. Having fixed $N$, choose $P$ sufficiently large so that $D_{1}<\epsilon, D_{3}<\epsilon, D_{4}<\epsilon$ for $p \geqq P$. It remains to justify these statements. For $D_{6}$ we use a result in a paper by J. D. Tamarkin [5]. For $D_{2}$ we use Lemma 2, and for $D_{4}$ and $D_{5}$ Lemma 1 . In $D_{1}$ and $D_{3}$ we are dealing essentially with integrals of continuous functions and the sums which tend to the integrals as limits, the number of terms being finite.

Now consider $u^{\text {(iv) }}-\left[\rho^{4}+\lambda(x)\right] u=0, \lambda(x)$ being an arbitrary continuous function. A fundamental system of solutions of $u^{\text {(iv) }}-\rho^{4} u=0$ is $u_{1}=\cos \rho x, u_{2}=\sin \rho x, u_{3}=e^{\rho x}, u_{4}=e^{-\rho x}$. By the method of variation of constants we have, as an equation satisfied by $u$,

$$
\begin{aligned}
u= & A \cos \rho x+B \sin \rho x+C e^{\rho x}+D e^{-\rho x} \\
& +\frac{1}{2 \rho^{3}} \int_{0}^{x} \lambda(t) u(t)\left[-\sin \rho(x-t)+\frac{e^{\rho(x-t)}}{2}-\frac{e^{-\rho(x-t)}}{2}\right] d t,
\end{aligned}
$$


where $A, B, C, D$ are arbitrary constants. Applying the boundary conditions of system $\mathrm{V}$ and choosing the multiplicative constant so that $A=1$, we have, assuming $\rho \neq 0$,

$u=\cos \rho x-\sin \rho x$

$$
\begin{gathered}
+\left[\operatorname { s i n } \rho x \left\{-2 e^{-\rho} \sin \rho+\frac{1}{2 \rho^{3}} \int_{0}^{1} \lambda(t) u(t)\left[-e^{-\rho} \sin \rho(1-t)\right.\right.\right. \\
\left.\left.-e^{-\rho} \cos \rho(1-t)+e^{-\rho t}\right] d t\right\} \\
+e^{-\rho(1-x)} \sin \rho-\frac{1}{4 \rho^{3}} \int_{0}^{1} \lambda(t) u(t)\left[-e^{-\rho(1-x)} \sin \rho(1-t)\right. \\
\left.-e^{-\rho(1-x)} \cos \rho(1-t)+e^{\rho(x-t)}\right] d t \\
-e^{-\rho x}+e^{-\rho(1+x)} \cos \rho+\frac{1}{4 \rho^{3}} \int_{0}^{1} \lambda(t) u(t)\left[-e^{-\rho(1+x)} \sin \rho(1-t)\right. \\
\left.\left.-e^{-\rho(1+x)} \cos \rho(1-t)+e^{-\rho(x+t)}\right] d t\right]\left\{1-e^{-\rho} \sin \rho-e^{-\rho} \cos \rho\right\}^{-1} \\
+\frac{1}{2 \rho^{3}} \int_{0}^{x} \lambda(t) u(t)\left[-\sin \rho(x-t)+\frac{e^{\rho(x-t)}}{2}-\frac{e^{-\rho(x-t)}}{2}\right] d t,
\end{gathered}
$$

and, as an equation satisfied by the characteristic numbers $\rho=\rho_{n}$, $\cos \rho=2 e^{-\rho}-e^{-2 \rho} \cos \rho$

$$
\begin{aligned}
& +\frac{\sin \rho}{2 \rho^{3}} \int_{0}^{1} \lambda(t) u(t)\left[e^{-\rho} \cos \rho(1-t)-\frac{e^{-\rho t}}{2}-\frac{e^{-\rho(2-t)}}{2}\right] d t \\
& +\frac{\cos \rho}{2 \rho^{3}} \int_{0}^{1} \lambda(t) u(t)\left[-e^{-\rho} \sin \rho(1-t)+\frac{e^{-\rho t}}{2}-\frac{e^{-\rho(2-t)}}{2}\right] d t \\
& +\frac{1}{4 \rho^{3}} \int_{0}^{1} \lambda(t) u(t)\left[\sin \rho(1-t)-\cos \rho(1-t)+e^{-\rho(1-t)}\right] d t \\
& -\frac{e^{-\rho}}{4 \rho^{3}} \int_{0}^{1} \lambda(t) u(t)\left[-e^{-\rho} \sin \rho(1-t)-e^{-\rho} \cos \rho(1-t)+e^{-\rho t}\right] d t .
\end{aligned}
$$

For each $\rho=\rho_{n}, u$ is a continuous function; we show $u$ uniformly bounded in $n$. In $u$, the terms

$$
\begin{gathered}
-\frac{1}{4 \rho^{3}} \int_{0}^{1} \lambda(t) u(t) e^{\rho(x-t)} d t\left\{1-e^{-\rho} \sin \rho-e^{-\rho} \cos \rho\right\}^{-1}, \\
\frac{1}{4 \rho^{3}} \int_{0}^{x} \lambda(t) u(t) e^{\rho(x-t)} d t
\end{gathered}
$$

may be combined to give 


$$
\begin{array}{r}
{\left[-\frac{1}{4 \rho^{3}} \int_{x}^{1} \lambda(t) u(t) e^{\rho(x-t)} d t-\frac{\sin \rho+\cos \rho}{4 \rho^{3}} \int_{0}^{x} \lambda(t) u(t) e^{-\rho(1-x+t)} d t\right]} \\
\cdot\left\{1-e^{-\rho} \sin \rho-e^{-\rho} \cos \rho\right\}^{-1} .
\end{array}
$$

For $t$ in $[x, 1], x-t \leqq 0$; for $t$ in $[0, x], 1-x+t \geqq 0$. Also, there exists a constant $c>0$ such that $1-e^{-\rho} \sin \rho-e^{-\rho} \cos \rho \geqq c$ for $\rho=\rho_{n}$. Call $M_{n}=\max _{[0,1]}\left|u_{n}(x)\right|, K=\int_{0}^{1}|\lambda(t)| d t$. Then

$$
\begin{gathered}
\left|u_{n}(x)\right| \leqq 2+\frac{5}{c}+\frac{17 M_{n} K}{4 c \rho_{n}^{3}}, \quad M_{n} \leqq 2+\frac{5}{c}+\frac{17 M_{n} K}{4 c \rho_{n}^{3}}, \\
M_{n} \leqq \frac{2+5 / c}{1-17 K /\left(4 c \rho_{n}^{3}\right)} .
\end{gathered}
$$

Thus for $n$ sufficiently great, $M_{n} \leqq 4+10 / c$. The remaining $n$ 's form a finite set. Hence $u_{n}(x)$ is uniformly bounded in $n$. Thus

$$
u_{n}(x)=\cos \rho_{n} x-\sin \rho_{n} x+e^{-\rho_{n}(1-x)} \sin \rho_{n}-e^{-\rho_{n} x}+r_{n}(x) / \rho_{n}^{3},
$$

and $\cos \rho_{n}=\phi\left(\rho_{n}\right)$, where $\lim _{n \rightarrow \infty} \phi\left(\rho_{n}\right)=0$. Thus $\rho_{n}=(n+1 / 2) \pi+\epsilon_{n}$, where $\lim _{n \rightarrow \infty} \epsilon_{n}=0$. It follows that

$$
\begin{aligned}
u_{n}(x)= & \cos (n+1 / 2) \pi x-\sin (n+1 / 2) \pi x \\
& +(-1)^{n} \exp \{-(n+1 / 2) \pi(1-x)\} \\
& -\exp \{-(n+1 / 2) \pi x\}+r_{n}(x) / n^{3} .
\end{aligned}
$$

The theorem for system $\mathrm{V}$ follows.

\section{REFERENCES}

1. C. M. Jensen, Some problems in the theory of interpolation by Sturm-Liouville functions, Transactions of this Society, vol. 29 (1927), pp. 54-79.

2. Rayleigh, Theory of Sound, vol. 1, London, Macmillan, 1929, pp. 275-276.

3. Dunham Jackson, Theory of Approximation, American Mathematical Society Colloquium Publications, vol. 11, New York, 1930, p. 134.

4. Stekloff and Tamarkin, Problème des vibrations transversales d'une verge élastique homogène, Rendiconti del Circolo Matematico di Palermo, vol. 31 (1911), pp. 341-362.

5. J. D. Tamarkin, Some general problems of the theory of ordinary linear differential equations and expansion of an arbitrary function in series of fundamental functions, Mathematische Zeitschrift, vol. 27 (1927), pp. 1-54.

Brown UNIVERSITy 